\title{
Refletindo sobre a integração dos sistemas fisiológicos: Avaliação de uma sequência didática fundamentada nos três momentos pedagógicos
}

\author{
Reflecting on the integration of physiological systems: Evaluation of a didactic sequence based on the \\ three pedagogical moments \\ Reflexionando sobre la integración de sistemas fisiológicos: Evaluación de una secuencia didáctica a \\ partir de los tres momentos pedagógicos
}

Recebido: 22/03/2021 | Revisado: 29/03/2021 | Aceito: 31/03/2021 | Publicado: 12/04/2021

Lúcia Maria de Almeida

ORCID: https://orcid.org/0000-0001-6435-0892 Centro Universitário Facex, Brasil E-mail: Imalmeida05@yahoo.com

Priscila Daniele Fernandes Bezerra Souza ORCID: https://orcid.org/0000-0002-2474-9193 Centro Universitário Facex, Brasil E-mail: prisciladani@yahoo.com.br Daniele Bezerra dos Santos

ORCID: https://orcid.org/0000-0001-7896-6946 Instituto Federal do Rio Grande do Norte, Brasil E-mail: Daniele.bezerra@ifrn.edu.br

Thatiane Brito da Silva Medeiros ORCID: https://orcid.org/0000-0002-6631-4155 Universidade Federal do Rio Grande do Norte, Brasil E-mail: thatybrito.rn@bol.com.br

Alvaro da Costa Freire

ORCID: https://orcid.org/0000-0002-2246-2068 Universidade Federal do Rio Grande do Norte, Brasil E-mail: alvarodcfreire@gmail.com

Clécio Danilo Dias da Silva

ORCID: https://orcid.org/0000-0002-7776-8830 Universidade Federal do Rio Grande do Norte, Brasil E-mail: danilodiass18@gmail.com

\begin{abstract}
Resumo
Considerando a relevância de trabalhar os conteúdos da fisiologia, explorando elementos que levem aos estudantes a compreenderem o funcionamento do organismo humano e a homeostase, o objetivo deste trabalho foi avaliar a opinião de estudantes do ensino médio sobre a estrutura, aplicação e eficácia de uma Sequência Didática (SD) explorando a integração dos sistemas fisiológicos, buscando assim sensibilizar a comunidade escolar com relação à saúde, as atitudes e os comportamentos, possibilitando o exercício da criticidade mediante ao processo de questionamento, formalização de conceitos e produção de conhecimentos. Foi utilizada a abordagem dos Três Momentos Pedagógicos. No primeiro momento, foram empregadas dinâmicas e questões contextualizadas envolvendo os temas selecionados. No segundo momento, foram desenvolvidas aulas dialógicas, rodas de conversas e leitura de materiais científicos sobre os sistemas fisiológicos explorados em aula. Durante o terceiro momento, foi efetivada a prática de "verificação de batimentos cardíacos e saturação". No final da aplicação da SD, os estudantes responderam a um questionário, que visou avaliar as atividades desenvolvidas. Os resultados obtidos por meio das ferramentas de avaliação demonstraram as potencialidades da SD proposta, e a relevância de se trabalhar os sistemas fisiológicos de forma integrada, de modo a possibilitar que os estudantes compreendam o funcionamento do corpo humano de forma significativa.
\end{abstract}

Palavras-chave: Fisiologia humana; Sequência didática; Três momentos pedagógicos; Educação básica.

\begin{abstract}
Considering the relevance of working on the contents of physiology, exploring elements that lead students to understand the functioning of the human organism and homeostasis the objective of this work was to evaluate the opinion of high school students on the structure, application and effectiveness of a Didactic Sequence (SD) exploring the integration of physiological systems, thus seeking to sensitize the school community regarding health, attitudes and behaviors , enabling the exercise of criticality through the process of questioning, formalization of concepts and production of knowledge. The Three Moments Pedagogical approach was used. In the first moment, dynamics and contextualized questions involving the selected themes were used. In the second moment, dialogic classes, conversation circles and
\end{abstract}


reading of scientific materials about the physiological systems explored in class were developed. During the third moment, the practice of "checking heart rate and saturation" was carried out. At the end of the application of the SD, the students answered a questionnaire, which aimed to evaluate the activities developed. The results obtained through the assessment tools demonstrated the potentialities of the proposed DS, and the relevance of working with the physiological systems in an integrated manner, in order to enable students to understand the functioning of the human body in a meaningful way.

Keywords: Human physiology; Following teaching; Three pedagogical moments; Basic education.

\section{Resumen}

Considerando la relevancia de trabajar los contenidos de la fisiología, explorando elementos que lleven a los estudiantes a comprender el funcionamiento del organismo humano y la homeostasis, el objetivo de este trabajo fue evaluar la opinión de estudiantes de secundaria sobre la estructura, aplicación y efectividad de una Secuencia Didáctica (DS) explorando la integración de sistemas fisiológicos, buscando así sensibilizar a la comunidad escolar en materia de salud, actitudes y conductas, posibilitando el ejercicio de la criticidad a través del proceso de cuestionamiento, formalización de conceptos y producción de conocimiento. Se utilizó el enfoque de los tres momentos pedagógicos. En un primer momento se utilizaron dinámicas y preguntas contextualizadas que involucran los temas seleccionados. En el segundo momento se desarrollaron clases dialógicas, círculos de conversación y lectura de materiales científicos sobre los sistemas fisiológicos explorados en clase. Durante el tercer momento se llevó a cabo la práctica de "comprobar la frecuencia cardíaca y la saturación". Al final de la aplicación de la SD, los estudiantes respondieron un cuestionario, que tenía como objetivo evaluar las actividades desarrolladas. Los resultados obtenidos a través de las herramientas de evaluación demostraron las potencialidades del SD propuesto, y la relevancia de trabajar con los sistemas fisiológicos de manera integrada, para que los estudiantes comprendan el funcionamiento del cuerpo humano de manera significativa.

Palabras clave: Fisiología humana; Siguiendo la enseñanza; Tres momentos pedagógicos; Educación básica.

\section{Introdução}

Independente do conteúdo abordado, inferimos que ensinar ciências de maneira atrativa, significativa e contextualizada é uma tarefa desafiadora para os docentes. No que se refere à aprendizagem do corpo humano, especificamente aos conteúdos voltados à morfologia e fisiologia, é visto que estes são abordados de maneira fragmentada por aparelhos e/ou sistemas, como se eles funcionassem de maneira isolada e individual. Essa situação torna-se um agravante pelo fato de os professores não promoverem a integração dos sistemas e suas funções em sala de aula, impossibilitando o discente a perceber a integridade existente entre os sistemas, e entender a dependência destes para o corpo funcionar como um todo (Silva, 2020).

Diante disto, algumas indagações tendem a surgir, tais como: "Qual a importância de se trabalhar o corpo humano e a integralização dos sistemas fisiológicos, considerando o contexto social e educacional que vivenciamos? Como relacionar e inserir esse conhecimento na vida dos discentes? Como atrair e estimular o interesse dos alunos a participar de forma mais ativa e significativa na construção desse conhecimento?"

Dessa maneira, Jesus (2014) afirma que a utilização de atividades lúdicas como encenações, jogos, modelização, elaboração de desenhos esquemáticos, uso de softwares educativos, leituras dinamizadas, e o uso de paródias podem ser empregados para facilitar a compreensão dos conteúdos de anatomia e fisiologia humana, assim como constatados por Ruppenthal (2013), Maturana e Costa (2013), Jesus (2014), e Guedes (2015). Além da utilização dessas metodologias alternativas, torna-se necessário o emprego de abordagens e de Sequências Didáticas (SD) que sejam capazes de aguçar a curiosidade dos alunos em relação aos conteúdos, permitindo que eles recebam as informações, questionem/problematizem estas informações, de modo a compreendê-las, reelabora-las, e aplicá-las em sua realidade cotidiana, corroborando para a manutenção da saúde e da qualidade de vida. Diante disto, destacamos a utilização da abordagem dos Três Momentos Pedagógicos (3MP), elaborada por Delizoicov (1991) e Delizoicov et al. (2002) e fundamentada nas concepções educacionais de Paulo Freire.

Embora os conteúdos relativos à morfofisiologia do corpo humano sejam abordados no ensino fundamental e no ensino médio, é perceptível que esses conhecimentos ainda são muito incipientes por parte dos alunos, visto que eles demonstram dificuldades em reconhecer tanto as estruturas como funcionamento dos órgãos e sistemas corporais, expressando também 
limitações em compreender e relacionar os sistemas e a sua funcionalidade de forma integrada para a manutenção da homeostase. Acredita-se que essa situação está relacionada à metodologia tradicional, mecanicista e memorística em que a temática vem sendo abordada por grande parte dos docentes na sua ação pedagógica. Nesse contexto, esse trabalho torna-se relevante por propor uma análise crítica e reflexiva de duas propostas que integram uma abordagem problematizadora, contextualizada e possível de ser utilizada no cotidiano dos discentes.

Considera-se que a abordagem dos (3MP) enfatiza o progresso no ensino e na aprendizagem de ciências de forma significativa, possibilitando ao aluno ressignificar e utilizar os conhecimentos relativos ao seu corpo e a sua saúde de forma reflexiva e modificar procedimentos e atitudes, para que o discente possa compreender as alterações que podem ocorrer em seu corpo com a passagem do tempo, por meio das mudanças de hábitos alimentares e mediante aos seus hábitos de vida. Essas mudanças de costumes dependem das necessidades do organismo, mediante a sua dependência e interação no meio em que vive, tais como: aalimentação, a higiene pessoal, o repouso adequado, entre outros que promovam a manutenção de uma vida saudável.

Este trabalho teve como objetivo avaliar a opinião de estudantes do ensino médio sobre a estrutura, aplicação e eficácia de uma Sequência Didática (SD) explorando a integração dos sistemas fisiológicos, buscando assim sensibilizar a comunidade escolar com relação à saúde, as atitudes e os comportamentos, possibilitando o exercício da criticidade mediante ao processo de questionamento, formalização de conceitos e produção de conhecimentos.

\section{Metodologia}

\subsection{Caracterização geral da pesquisa}

A presente pesquisa foi efetivada em uma escola pública na Cidade de Natal - RN, com 30 estudantes do $1^{\circ}$ ano do Ensino Médio, no período de agosto à novembro de 2018. Considerando o caráter dos processos metodológicos utilizados em consonância com os objetivos traçados (aplicação e avaliação de uma sequência de ensino), foi utilizada a abordagem quantiqualitativa com procedimentos direcionados a aplicação de questionários (Flick, 2009; Rosa, Oliveira, Orey, 2015). De acordo com Rosa, Oliveira e Orey (2015), atualmente, a abordagem quanti-qualitativa se constitui como uma tendência metodológica crescente em investigações na área de ensino, permitindo aos pesquisadores entenderem e compreenderem, de uma maneira holística, os problemas complexos enfrentados pela sociedade. Flick (2009) afirma que a convergência dos métodos quantitativos e qualitativos proporcionam mais credibilidade e legitimidade aos resultados encontrados, evitando o reducionismo à apenas uma opção.

Com relação às contribuições da pesquisa quanti-qualitativa, o autor destaca que esta: reúne o controle de vieses (métodos quantitativos) com compreensão, a partir dos agentes envolvidos na investigação (métodos qualitativos); agrega a identificação de variáveis específicas (métodos quantitativos), com uma visão global do fenômeno (métodos qualitativos); enriquece constatações obtidas sobre condições controladas com dados obtidos dentro do contexto natural de sua ocorrência; e permite a validade da confiabilidade das descobertas pelo emprego de técnicas diferenciadas.

\subsection{Sequência didática com base nos momentos pedagógicos}

Visando possibilitar uma compreensão sistemática dos sistemas fisiológicos para os estudantes envolvidos no processo formativo, fez-se necessário elaborar uma sequência de atividades que possibilitasse abordagem dos conteúdos em etapas de ensino e que conduzissem os discentes à reflexão e ao entendimento da temática, que foi mediada por estratégias didáticas, as quais proporcionam um processo de ensino-aprendizagem, a nosso ver, considerado como mais significativo. Nesse contexto, o trabalho teve como base a proposta educacional os Três Momentos Pedagógicos, conforme os pressupostos delineados por Delizoicov, Angotti e Pernambuco (2002, 2011). 
O primeiro contato com a turma se deu por intermédio da professora da disciplina na turma participante. Em seguida foi concedida a oportunidade para que ocorresse a interação dos pesquisadores com a turma. O Primeiro Momento Pedagógico foi realizado por meio de uma problematização inicial em que, a priori, foi efetivada uma sondagem com os alunos sobre situações vivenciadas em seu cotidiano e que se relacionam à temática. Essa sondagem foi feita utilizando-se de dinâmicas associadas a questões contextualizadas (por que depois de comer muito sentimos sono e preguiça? qual a relação de exercícios físico (malhar) com engordar e emagrecer? por que o coração acelera quando a gente corre ou se assusta?), bem como a assuntos já abordados em sala de aula e que também estão relacionados à temática. Assim, os alunos foram desafiados a exporem os seus pensamentos sobre situações do cotidiano.

Logo em seguida foi distribuído para cada aluno da turma um copo de $100 \mathrm{ml}$ de chá e uma torradinha, acompanhado das seguintes perguntas chave: "Quais partes do corpo e quais órgãos estão trabalhando neste momento para digerir o chá e a torrada que você comeu? Qual é a relação entre o intestino e o sangue? Por que o chá ingerido não sai junto com as fezes?". Partindo desses questionamentos iniciais, foram levantadas outras questões, buscando proporcionar momentos de reflexão para que os estudantes associassem as questões levantadas com os órgãos do sistema digestório e circulatório. Posteriormente, a turma foi dividida em grupos, para a realização de uma atividade. Nesta, os estudantes foram instruídos a observar uma imagem do aparelho digestório e tentar reconhecê-lo, citando os nomes de suas estruturas e respectivas funções.

No Segundo Momento Pedagógico, a organização do conhecimento ocorreu por meio de aulas expositivas e dialógicas, bem como por rodas de conversa e outras discussões que foram realizadas com o auxílio de um retroprojetor. Também foram utilizadas leituras de materiais científicos, artigos de revistas e leitura de mapas mentais que envolveram os temas trabalhados. Durante essas aulas foram explorados os conhecimentos envolvendo o "Sistema Digestório", o "Sistema Circulatório" e o "Sistema Respiratório" (funções; órgãos e musculatura, doenças associadas etc.), os quais podem ser observados no Quadro 1. Durante todas as etapas da organização do conhecimento foram explorados, na sala de aula, a respeito de como os sistemas abordados trabalhavam de forma integrada com outros órgãos e sistemas. Também eram discutidas as doenças que comumente são associadas a cada sistema, apresentando-as de forma específica e integrada.

Quadro 1: Conteúdos e estratégias explorados/utilizados na organização do conhecimento.

\begin{tabular}{|c|c|}
\hline CONTEÚDOS EXPLORADOS & ESTRATÉGIAS UTILIZADAS \\
\hline Órgãos, estruturas, funções e funcionamento do Sistema Digestório. & Aulas dialógicas \\
\hline Doenças envolvendo o sistema digestório & $\begin{array}{c}\text { Aulas dialógicas e leitura de Material } \\
\text { científico }\end{array}$ \\
\hline Órgãos, estruturas, funções e funcionamento do Sistema Circulatório. & Aulas dialógicas \\
\hline Doenças envolvendo o Sistema Circulatório & $\begin{array}{c}\text { Aulas dialógicas e leitura de Material } \\
\text { científico }\end{array}$ \\
\hline Integração do Sistema Digestório e Respiratório & Aulas dialógicas e Rodas de conversas \\
\hline Órgãos, estruturas, funções e funcionamento do Sistema Respiratório. & Aulas dialógicas \\
\hline Doenças envolvendo o Sistema Respiratório & $\begin{array}{c}\text { Aulas dialógicas e leitura de Material } \\
\text { científico }\end{array}$ \\
\hline Integração do Sistema Respiratório e Circulatório & Aulas dialógicas e Rodas de conversas \\
\hline
\end{tabular}

Fonte: Autores (2021).

Durante o Terceiro Momento Pedagógico houve a aplicação do conhecimento, onde foi realizada uma prática a respeito do funcionamento integrado da circulação e da respiração, a partir da "verificação de frequência respiratória e cardíaca". 
Inicialmente, por meio de um oxímetro, foram medidos os batimentos cardíacos e a saturação dos estudantes em estado de repouso, dentro da sala de aula. Os dados coletados individualmente foram anotados pelos alunos em seus respectivos cadernos. Posteriormente, os estudantes foram levados até a quadra da escola e foram instruídos a darem seis voltas correndo. Ao final, estes se encaminhavam para o ponto de concentração, onde eram verificados novamente a saturação e o batimento cardíaco individualmente. Depois de realizarem a contagem dos BPM (batimentos por minuto) e a saturação, os alunos compararam os resultados obtidos no momento em repouso e com aqueles que obtiveram após a realização de atividade física. Partindo disso, foi esclarecida aos estudantes a necessidade do corpo em sobrecarregar a respiração durante os exercícios, e o motivo do corpo apresentar alguns sinais vitais alterados, tais como batimentos elevados, respiração ofegante, cansaço e sede.

\subsection{Avaliação da sequência didática}

Ao final da aplicação da SD, os educandos foram convidados a avaliar a metodologia empregada na pesquisa e suas contribuições para o processo de aprendizagem dos conteúdos abordados. Para isso, utilizamos como instrumento de coleta um questionário investigativo, do tipo "escala de Likert", que permite mensurar o nível de aprendizagem dos investigados por meio da metodologia empregada. Este material apresentava 05 afirmações acerca das atividades desenvolvidas (Quadro 2) e um elenco de sentenças para as quais os sujeitos da pesquisa pudessem manifestar o seu grau de concordância, assinalando: Concordo (C); Não Concordo (NC); Indiferente (IN). Segundo Zanella, Seidiel e Lopes (2010), este recurso é comumente utilizado em levantamentos de atitudes, opiniões e avaliações, e tem contribuído de maneira significativa, agregando confiabilidade em pesquisas na área da educação. De modo geral, os dados obtidos foram agrupados e categorizados em planilhas no aplicativo Microsoft Excel 2010, para a elaboração de gráficos e tabelas, de modo a delinear a construção dos resultados e das discussões deste estudo.

\section{Resultados e Discussão}

Sabe-se que a aplicação e a avaliação de estratégias metodológicas diversificadas e/ou sequências de ensino empregadas durante o processo de ensino e aprendizagem é um excelente aliado para os professores no âmbito educacional. É nesse contexto que Ferreira e Dias-da-Silva (2017) chamam atenção para a realização de avaliações a respeito das metodologias utilizadas em sala de aula. A partir dessas avaliações é possível analisar se a metodologia está dando certo no ambiente de estudo, trazendo um "feedback" acerca destas, possibilitando aos docentes a possibilidade de melhoria de suas práticas pedagógicas no contexto escolar. Nesse sentido, visando conhecer a opinião destes sobre a estrutura, aplicação e eficácia da SD proposta para abordar a integração dos sistemas fisiológicos no ensino médio, analisamos as respostas dos alunos, obtidas por meio das afirmativas presentes no instrumento de avaliação (Quadro 2). 
Quadro 2: Ferramenta de avaliação da Sequência de ensino utilizada.

\begin{tabular}{|c|c|c|c|}
\hline AFIRMATIVAS & $\mathbf{C}$ & NC & IN \\
\hline 1. Eu nunca estudei os sistemas do corpo humano de forma integrada & $76 \%$ & $15 \%$ & $9 \%$ \\
\hline $\begin{array}{l}\text { 2. Os questionamentos realizados no início das atividades eram contextualizados com o meu } \\
\text { cotidiano e, juntamente com os conceitos discutidos em sala de aula, me motivaram a refletir } \\
\text { sobre os temas trabalhados. }\end{array}$ & $88 \%$ & $0 \%$ & $12 \%$ \\
\hline $\begin{array}{l}\text { 3. As estratégias didáticas diversificadas e dinâmicas de ensino utilizadas contribuíram para uma } \\
\text { aprendizagem mais satisfatória e significativa dos conteúdos envolvendo os sistemas } \\
\text { fisiológicos. }\end{array}$ & $100 \%$ & $0 \%$ & $0 \%$ \\
\hline $\begin{array}{l}\text { 4. As aulas dialógicas e rodas de conversas durante a organização dos conhecimentos } \\
\text { possibilitaram a minha compreensão dos conteúdos envolvendo os sistemas trabalhados. }\end{array}$ & $73 \%$ & $21 \%$ & $6 \%$ \\
\hline $\begin{array}{l}\text { 5. As atividades desenvolvidas em sala de aula utilizadas para aplicar os conhecimentos } \\
\text { envolvendo os sistemas fisiológicos de forma integrada me possibilitaram melhor compreender } \\
\text { como funciona o corpo humano. }\end{array}$ & $100 \%$ & $0 \%$ & $0 \%$ \\
\hline
\end{tabular}

Legenda: C (Concordo), NC (Não Concordo), IN (Indiferente). Fonte: Autores (2021).

No que diz respeito à primeira afirmativa, $76 \%$ dos estudantes concordam que "Eu nunca estudei os sistemas do corpo humano de forma integrada", 15\% dos alunos não concordam com a afirmação e 9\% dos discentes demonstraram-se indiferente quanto à assertiva. Conforme Ferreira, Coutinho e Silva (2002) são fundamentais o desenvolvimento de uma visão sistêmica dos seres humanos e dos seres vivos em geral, pois o organismo funciona como um todo. Entretanto, os dados obtidos demostraram que a abordagem fragmentada dos conteúdos da fisiologia ainda hoje é uma situação comum de se encontrar nas aulas de Ciências e Biologia na educação básica. Conforme discutido por Vanzela, Balbo, Della-Justina (2013) e Maturana e Costa (2013), essa situação torna-se um agravante, pois a exploração isolada dos sistemas fisiológicos impossibilita aos discentes perceberem a integridade existente entre as estruturas e os órgãos dos aparelhos, e a entenderem a dependência destes para o corpo funcionar de forma equilibrada.

Na segunda afirmativa, constatamos que $88 \%$ dos estudantes concordam que "Os questionamentos realizados no início das atividades eram contextualizados com o meu cotidiano e, juntamente com os conceitos discutidos em sala de aula, me motivaram a refletir sobre os temas trabalhados", e 12\% assinalaram a sentença Indiferente. Levando-se em consideração os dados obtidos, percebe-se a importância do momento de problematização inicial, os quais foram norteados pela dialogicidade e questões problematizadoras, o que na visão dos estudantes contribuíram no processo de ensino e aprendizagem, tornando o percurso da construção do conhecimento mais significativo. Nesse contexto, Freire (1996), assegura que é necessário conhecer e respeitar os saberes dos alunos, advindos das experiências anteriores a sala de aula e de suas realidades, a fim de envolvê-los no processo de ensino-aprendizagem. De acordo com Gehlen (2009) é através da problematização que os estudantes são desafiados a expor os seus entendimentos sobre determinadas situações significativas, conceitos e entendimentos sobre a temática abordada. É por meio deste momento que o docente consegue identificar os conhecimentos prévios que os alunos apresentam, e assim ele pode adequar seu planejamento de ensino de acordo com as necessidades de aprendizagem da turma, possibilitando também acompanhar e avaliar os progressos dos educandos durante as suas atividades de ensino (Delizoicov; Angotti; Pernambuco, 2002, 2011). É importante salientar que este momento inicial de problematização é extremamente relevante, uma vez que ele permite instigar o aluno para participar da discussão e reflexão, fazendo com que sinta a necessidade de adquirir outros conhecimentos, linguagens e comportamentos (Dias-da-Silva et al., 2016; Silva, 2020).

Para a terceira afirmativa, verificamos que 100\% dos alunos concordam que "As estratégias didáticas diversificadas e dinâmicas de ensino utilizadas contribuíram para uma aprendizagem mais satisfatória e significativa dos conteúdos envolvendo os sistemas fisiológicos". Considerando que os sistemas fisiológicos envolvem uma diversidade de terminologias e funções 
entorno dos diversos órgãos e estruturas, os conteúdos da fisiologia muitas vezes são classificados como de difícil apreensão pelos estudantes. Nesse contexto, Segundo Sasseron (2013) e Almeida, Dias-da-Silva e Santos (2018), compete ao professor procurar métodos de ensino que facilitem a compreensão dos estudantes como forma de dinamizá-lo. Corroborando com este pensamento, Jesus (2014) afirma que a utilização de atividades lúdicas como encenações, jogos, modelização, elaboração de desenhos esquemáticos, uso de softwares educativos, leituras dinamizadas, e o uso de paródias podem ser empregados para facilitar a compreensão dos conteúdos de anatomia e fisiologia humana.

No que diz respeito a quarta afirmativa, $73 \%$ dos alunos concordam que "As aulas dialógicas e rodas de conversas durante a organização dos conhecimentos possibilitaram a minha compreensão dos conteúdos envolvendo os sistemas trabalhados", 21\% discentes não concordam e $6 \%$ dos estudantes demonstraram-se indiferentes à afirmação. Verifica-se por meio desses dados que a maioria dos estudantes consideram relevante o uso das aulas dialógicas e rodas de conversas para a construção dos conhecimentos explorados na SD. Assim, torna-se importante destacar que, as atividades envolvendo diálogos e discussões promovem o desenvolvimento cognitivo do aluno, bem como contribuem para a organização e, consequentemente, com a aprendizagem dos conteúdos da biologia, que auxiliam os estudantes a lidar com as informações, compreendê-las e reelaborá-las, e assim compreender e interagir com o mundo e nele agir com autonomia. Ainda com relação à organização do conhecimento, Dias-da-Silva et al. (2018) ressalta a relevância da mediação por parte dos professores em todo o percurso formativo, uma vez que os aprendentes, constantemente, trazem novos questionamentos, diferentes daqueles utilizados na problematização inicial, levando aos professores a inserir os conceitos científicos, conforme a demanda percebida no ambiente de aprendizagem. De acordo com Nascimento et al. (2018), como mediador da aprendizagem, o professor necessita contextualizar a fundamentação teórica com a prática, para uma ação mais coerente com a realidade da sala de aula. Dessa forma, este momento pedagógico exige um maior cuidado e atenção por parte dos docentes, visto que estes precisam dialogar com os discentes, e a partir deste diálogo, explorar informações e contextos da realidade deles, o que possibilitará selecionar melhor conteúdos e métodos a serem trabalhados, de forma a atender as necessidades de aprendizagem verificadas na turma em sua complexa heterogeneidade.

No que se refere à quinta e última afirmativa, 100\% dos estudantes concordam que "As atividades desenvolvidas em sala de aula utilizadas para aplicar os conhecimentos envolvendo os sistemas fisiológicos de forma integrada me possibilitaram melhor compreender como funciona o corpo humano". Esses dados são significativos pois evidenciam a relevância de atividades diversificadas dentro da etapa de aplicação do conhecimento, em especial aquelas que proporcionem uma aprendizagem ativa dos estudantes, como é o caso da prática de verificação de frequência respiratória e utilizada na SD aplicada. Conforme e Gehlen, Maldaner e Delizoicov (2012), a aplicação de conhecimentos destina-se a empregar o conhecimento do qual o estudante vem se apropriando para analisar e interpretar as situações propostas na problematização inicial e outras que possam ser explicadas e compreendidas pelo mesmo corpo de conhecimentos. Para esses autores, nessa etapa, o papel do professor consiste em desenvolver diversas atividades para capacitar os alunos a utilizarem os conhecimentos científicos explorados na organização do conhecimento, com a perspectiva de formá-los para articular constantemente a conceituação científica com situações que fazem parte de sua vivência.

\section{Considerações Finais}

Considerando a necessidade de se trabalhar conteúdos de forma contextualizada com a realidade cotidiana do aluno, na qual estes conhecimentos possam fazer sentido na sua vida diária e intervir na sua realidade, principalmente conteúdos de fisiologia humana que envolvem aspectos culturais e sociais, a abordagem dos três momentos pedagógicos explorando a integração dos sistemas fisiológicos possibilitou aos discentes discutir e aprofundar questões relacionadas a saúde, hábitos e comportamentos de forma crítica e reflexiva. Esse fato pode ser constatado visto que para a maioria dos educandos a SD utilizada 
proporcionou uma participação mais efetiva nas aulas, uma reflexão a partir da introdução de uma problematização e consequente discussão, a tomada de consciência da necessidade de pesquisa, leituras e organização dos conhecimentos e suas possibilidades de aplicação/utilização no seu cotidiano, gerando uma aprendizagem mais satisfatória. Assim, pode-se inferir que a SD utilizada dentro da abordagem dos 3MP corroborou com processo formativo dos estudantes acerca da temática. Ressalta-se ainda a importância de pesquisas que possam contribuir para melhorar o processo de ensino e aprendizagem envolvendo a temática de fisiologia humana, considerando os contextos históricos, sociais e culturais da comunidade escolar.

Compreender como os sistemas corporais funcionam de forma integrada é um objetivo não muito simples de alcançar, principalmente quando são utilizados no processo de ensino-aprendizagem métodos fundamentados em uma didática de caráter tradicional. Nesse contexto, diante dos resultados obtidos, pode ser observado as potencialidades de uma sequência didática envolvendo questões problematizadoras, rodas de conversas, dinâmicas e atividades práticas para se trabalhar conteúdos integrados de fisiologia na educação básica.

Salienta-se que, sem estratégias adequadas, torna-se difícil proporcionar momentos e situações para que os estudantes consigam entender os processos fisiológicos do corpo humano, os quais, muitas vezes, são considerados como complexos por grande parte dos alunos para aprender e pelos professores para ensinar. Dessa forma, partindo da opinião dos educandos podese afirmar que a abordagem dos três momentos pedagógicos possibilitou melhorar o processo de ensino e aprendizagem da fisiologia, uma vez que, esta abordagem utiliza os conteúdos numa perspectiva dialógica e problematizadora, possibilitando aos discentes a participar de forma ativa do processo de construção do conhecimento, conjecturar a partir de questionamentos de situações reais de do cotidiano, além de pesquisar e reelaborar conhecimentos através de suas concepções prévias.

\section{Referências}

Almeida, L. M., Dias-da-Silva, C. D., \& Santos, D. B. (2018). Educação para a saúde e sexualidade: uma abordagem metodológica a partir dos questionamentos dos discentes. In: Araújo, R. M., Pinheiro, R. R. L. M. P. (Org.). Educação, Direitos Humanos, Cidadania e Gestão. Natal: Unifacex.

Delizoicov, D. (1991). Conhecimento, tensões e transições. 214 f. Tese (Doutorado) - Faculdade de Educação, Universidade de São Paulo, São Paulo.

Delizoicov, D., Angotti, J., \& Pernambuco, M. M. (2011). Ensino de Ciências: fundamentos e métodos. (4a ed.), Cortez.

Dias-da-Silva, C. D., Silva, G. F., Almeida, L.M., Almeida, R. G. \& Souza, R. G. (2018). Abordando o sistema respiratório em uma perspectiva dos três momentos pedagógicos. CARPE DIEM: Revista Cultural e Científica do UNIFACEX, 16(1), 29-43.

Dias-da-Silva, C. D., Cavalcante, B.P., Maciel, L.G.B., Santos, D.B., \& Almeida, L.M. (2016). Aprendendo sobre o corpo humano: contribuições do PIBID para o ensino de ciências. CARPE DIEM: Revista Cultural e Científica do UNIFACEX. 14(1), 17-30.

Ferreira, N. P., \& Dias-da-Silva, C. D. (2017). Praticas educativas no ensino de Ciências e Biologia: propostas didáticas para a educação básica. Alemanha: Novas edições Acadêmicas.

Ferreira, V., Coutinho, F., Silva, F. (2002). Concepções alternativas ou perfis conceituais? Um estudo com educadores em ciências sobre a função digestiva. In: Encontro "Perspectivas do ensino de Biologia", 8. São Paulo. Anais. São Paulo.

Flick, U. (2009). Uma introdução à pesquisa qualitativa. (4a ed.), Bookman.

Freire, P. (1996). Pedagogia da Autonomia - Saberes necessários à prática educativa. Paz e Terra.

Gehlen, S. T, Maldaner, O. A., \& Delizoicov, D. (2012). Momentos pedagógicos e as etapas da situação de estudo: complementaridades e contribuições para a educação em ciências. Ciência \& Educação, 18(1), 1-22.

Gehlen, S. T. (2009). A função do problema no processo ensino-aprendizagem de ciências: contribuições de Freire e Vygotsky. 253 f. Tese (Doutorado em Educação Científica e Tecnológica) - Universidade Federal de Santa Catarina, Florianópolis.

Guedes, M. R. A. (2015). Ensino de anatomia e fisiologia do sistema digestório humano mediado por sala ambiente, 72 f. Dissertação (mestrado), Centro Universitário de Volta Redonda, RJ.

Jesus, L. R. (2014). Ensinando o sistema circulatório no ensino fundamental. 135 f. Dissertação (mestrado em educação), Universidade de São Paulo, SP.

Maturana, L. G., \& Costa, J. R. S. (2013). Anatomia humana como proposta prático-pedagógica para aplicar o tema transversal saúde na rede estadual de ensino de Diamantina - MG. Revista Vozes dos Vales da UFVJM, 5(2),01-13. 
Research, Society and Development, v. 10, n. 4, e30610414170, 2021

(CC BY 4.0) | ISSN 2525-3409 | DOI: http://dx.doi.org/10.33448/rsd-v10i4.14170

Nascimento, A. C. L. M., Dias-da-Silva, C. D., Santos, D.B., Silva, L.E.O., Almeida, L.M., \& Carvalho França, N.N. (2018). Atividades práticas no ensino de ciências: a relação teoria e prática e a formação do licenciando em ciências biológicas. CARPE DIEM: Revista Cultural e Científica do UNIFACEX, 16(1), 4460.

Pagel, U. R., Campos, L. M, \& Batitucci, M. C. P. (2015). Metodologias e práticas docentes: uma reflexão acerca da contribuição das aulas práticas no processo de ensino-aprendizagem de biologia. Experiências em Ensino de Ciência.s 10(2), 4-25.

Rosa, M., Oliveira, D. P. A., \& Orey, D. C. (2015). Delineando e Conduzindo o Método Misto de Pesquisa em Investigações em Educação Matemática. Perspectivas em Educação Matemática, 8(3), 749-769.

Silva, C. D. D. (2020). Ciências biológicas: realidades e virtualidades. Atena Editora.

Sasseron, L. H. (2013). Interações discursivas e investigação em sala de aula: o papel do professor. Cengage Learning.

Vanzela, E. C., Balbo, S.L., \& Della-Justina, L. A. A. (2013). Integração dos sistemas fisiológicos e sua compreensão por alunos do nível médio. Arquivos do Museu Dinâmico Interdisciplinar, 11(3), 12-19.

Zanella, A., Seidel, E. J., \& Lopes, L. F. D. (2010). Validação de questionário de satisfação usando análise fatorial. Revista de Inovação, gestão e Produção, 2(12), 102-112. 